\title{
LOCATION OF EARTHQUAKE SWARM EVENTS NEAR PALMDALE, CALIFORNIA, USING A LINEAR GRADIENT VELOCITY MODEL
}

\author{
By Dayna Salter Drowley and Karen C. McNally
}

\begin{abstract}
A series of small earthquakes $\left(0.5 \leqq M_{L} \leqq 3.0\right)$ along a $60-\mathrm{km}$ segment of the San Andreas Fault in the vicinity of Palmdale, California, has been recorded since 1976 by an array operated by the California Institute of Technology. The events were analyzed in two steps. First, travel-time data from four regionally well-recorded events $\left(M_{L}=2.2,2.8,3.0,2.8\right)$ were inverted using a nonlinear least-squares algorithm to obtain a local velocity model consisting of an upper crustal layer with linearly increasing velocity in dipping contact with a constant velocity half-space. Hypocenters of over 150 events were relocated using this velocity model. Most of the events are clustered between the mapped traces of the San Andreas and Punchbowl faults; however, there has been a migration of activity along the San Andreas Fault. Activity which began in a 5-km cluster has expanded during a $2-\mathrm{yr}$ period to fill a $60-\mathrm{km}$ segment of the fault.
\end{abstract}

\section{INTRODUCTION}

More than 150 small earthquakes $\left(0.5 \leqq M_{L}<3.0\right)$ have occurred as a prolonged sequence near the San Andreas Fault in the vicinity of Palmdale, California (Figure 1) since 1976. The occurrence of this series of earthquakes is of considerable interest by virtue of the documented $47 \mathrm{yr}$ of seismic quiescence (Hileman et al., 1973; Friedman et $a l ., 1976$ ) in this area (which last broke during the great earthquake of 1857) and its proximity to the observed uplift known as the Palmdale "bulge" (Castle et al., 1976). Other features of the swarm, such as time-dependent rotation of stress axes inferred from focal mechanisms, have been described elsewhere ( $\mathrm{McNally}$ et al., 1978).

The California Institute of Technology and the U.S. Geological Survey jointly operate an extensive network of short period vertical seismographic stations in southern California. In addition to the permanent network, a mobile array of seismographic trailers was installed around the swarm area in 1976 to 1978 (Figure 2). Thus, the arrival times for the events of this swarm were well recorded by a dense local network. Arrival times were read to the nearest $0.01 \mathrm{sec}$, however, errors in reading emergent arrivals were probably of the order 0.05 to $0.10 \mathrm{sec}$.

The goal of the study presented herein is a detailed examination of temporal and spatial patterns of occurrence in the swarm. Relatively precise hypocentral locations necessary in such studies depend upon an accurate velocity model for the upper crust in the region of the swarm. The preliminary locations of the swarm events had been based on the average southern California regional model which consists of constant velocity layers. In this study, an upper crustal model was estimated for the local region of the swarm using $P$-wave travel-time data from four well-recorded events. The new velocity model, consisting of an upper crustal layer having linearly increasing velocity model, with depth in dipping contact with a constant velocity half-space, was used to relocate all events in the swarm. 


\section{The Method}

The model for the upper part of the crust that was used in this study is shown schematically in Figure 3. The parameters of the model are
$v_{0}=$ initial layer velocity
$k=$ velocity gradient in layer
$v_{h}=$ half-space velocity
$H_{0}=$ depth to half-space at reference station
$a=$ latitude dip parameter $(\mathrm{km} / \mathrm{deg})$

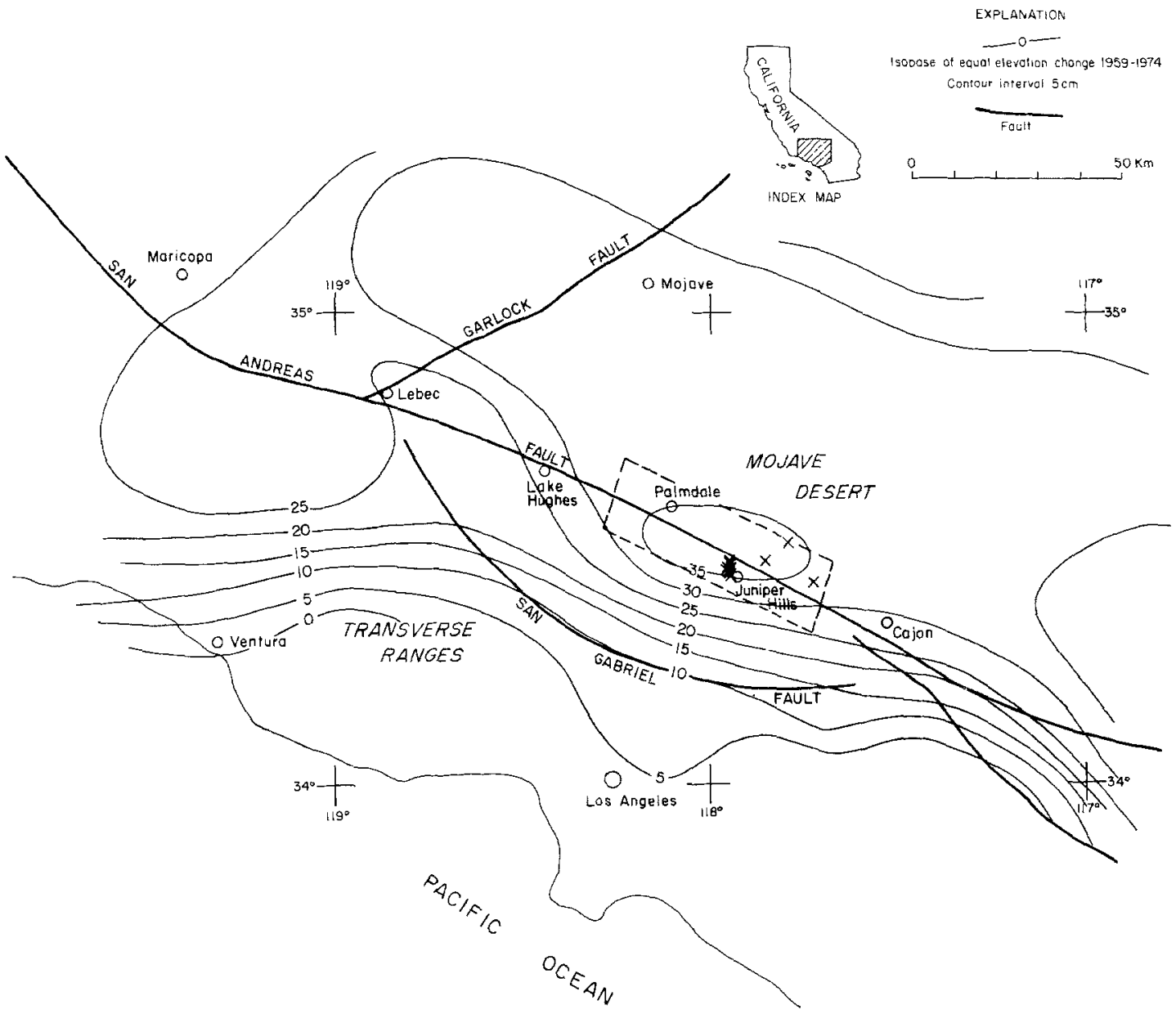

FIG. 1. Location of the swarm area Juniper Hills relative to major features in southern California.

$b=$ longitude dip parameter $(\mathrm{km} / \mathrm{deg})$

where the depth to the half-space at any point having latitude $\phi$ and longitude $\lambda$ is

$$
\begin{aligned}
& z=H_{0}+a \Delta \phi+b \Delta \lambda \\
& \text { where } \Delta \phi=\phi-\phi_{\text {ref }} \\
& \Delta \lambda=\lambda-\lambda_{\text {ref }} \\
& z_{r}=\text { depth to half-space under receiver } \\
& z_{s}=\text { depth to half-space under source. }
\end{aligned}
$$


The simplicity of a gradient velocity layer over a constant velocity half-space allows the use of an analytical travel-time function.

Following McEvilly (1966), the travel-time function for the direct ray is

$$
t=\frac{1}{k}\left[\cosh ^{-1}\left(\frac{v_{m}}{v_{0}+k d}\right)-\cosh ^{-1}\left(\frac{v_{m}}{v_{0}}\right)\right]
$$

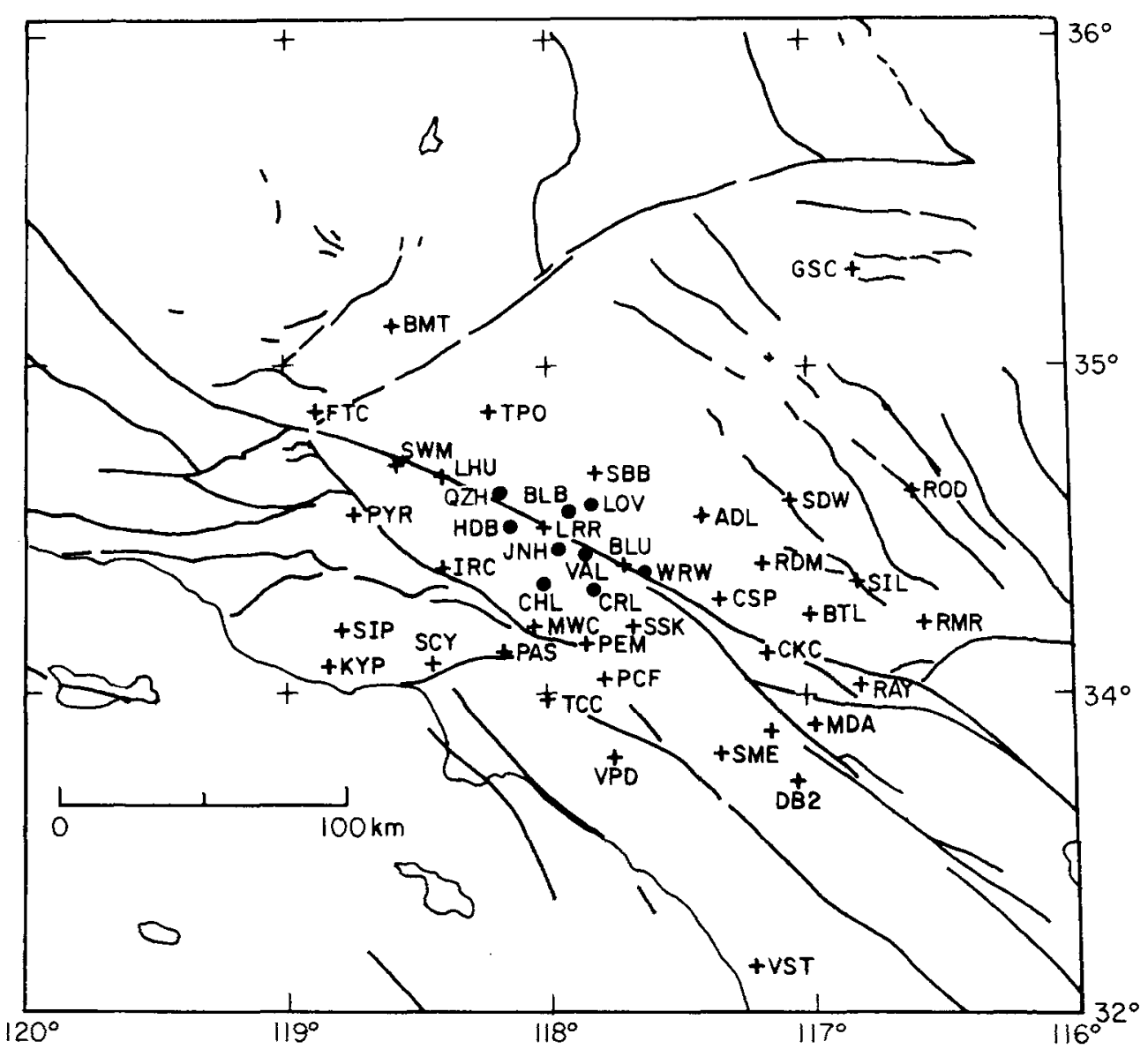

FIG. 2. Permanent stations of the southern California Seismic Network (SCARLET) $(+) 1$ and trailer array stations $\left(^{*}\right)$ used in the velocity model estimation.

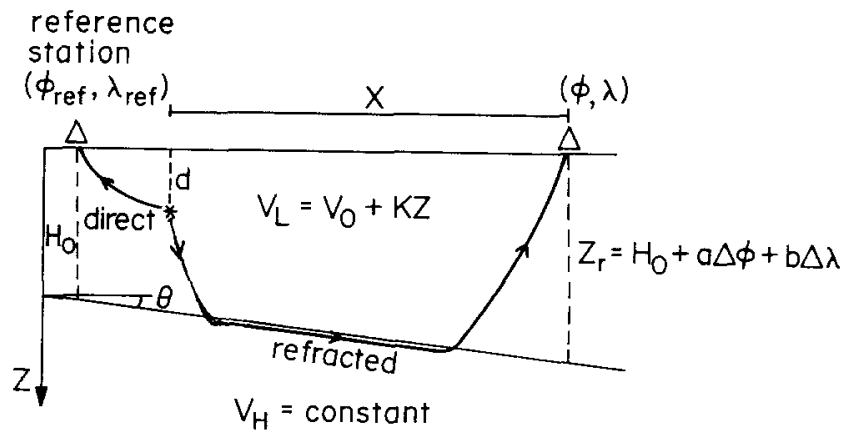

FIG. 3. Schematic diagram of the velocity model used in the study. The parameters are described in the text. 
and for refracted ray, assuming the dip in the layer half-space is small, the traveltime function is

$$
\begin{aligned}
t=\frac{X}{v_{h}} \cos \theta+\frac{1}{k}\left[\cosh ^{-1}\left(\frac{v_{m}}{v_{0}+k d}\right)+\cosh ^{-1}\left(\frac{v_{m}}{v_{0}}\right)-\cosh ^{-1}\left(\frac{v_{m}}{v_{0}+k z_{s}}\right)\right. \\
\left.-\cosh ^{-1}\left(\frac{v_{m}}{v_{0}+k z_{r}}\right)\right]+\frac{1}{k}\left\{\left[1-\left(\frac{v_{0}+k z_{s}}{v_{h}}\right)^{2}\right]^{1 / 2}+\left[1-\left(\frac{v_{0}+k z_{r}}{v_{h}}\right)^{2}\right]^{1 / 2}\right. \\
\left.-\left[1-\left(\frac{v_{0}+k d}{v_{h}}\right)^{2}\right]^{1 / 2}-\left[1-\left(\frac{v_{0}}{v_{h}}\right)^{2}\right]^{1 / 2}\right\}
\end{aligned}
$$

where $v_{0}, k, v_{h}, H_{0}, z_{r}$, and $z_{s}$ are as defined above

$v_{m}=$ the velocity at the bottom of a ray which for the refracted ray is $v_{h}$

$X=$ the distance between the epicenter and station recording a refracted arrival

$d=$ earthquake depth

$\theta=$ angle of apparent dip of the layer half-space interface (source to station).

Both the velocity model estimation problem and the hypocenter determination problem may be formulated by linearizing the travel-time function using a first order expansion. Then, small changes in the time function are related to small changes in the model through

$$
\Delta \underline{t}=\underline{\underline{A}} \Delta \underline{m}
$$

where $\Delta \underline{t}$ is the residual vector composed of observed arrival times minus calculated arrival times. $A$ contains the analytic partial derivatives of the travel-time function with respect to the model parameters, and $\Delta \underline{m}$ is the solution vector. In the velocity model estimation problem, $\Delta \underline{m}$ contains corrections to the velocity and structure parameters, and in the hypocenter determination problem, $\Delta \underline{m}$ contains corrections to the hypocentral parameters.

For the usual least-squares problem, existence of a solution depends on the nonsingularity of $\underline{\underline{A}}^{T} \underline{\underline{A}}$. In this study, the Levenberg-Marquardt nonlinear leastsquares algorithm (Levenberg, 1944; Marquardt, 1963; Hoerl and Kennard, 1970) was used to estimate the solution vector $\underline{m}$. The Levenberg-Marquardt algorithm ensures the nonsingularity of $\underline{\underline{A}}^{T} \underline{\underline{A}}$ so that a solution will always exist. The algorithm works by adding a factor, $K$, to the diagonal elements of $\underline{\underline{A}}^{T} \underline{\underline{A}}$. The effect of the factor $K$ is to taper the eigenvalue spectrum of $\underline{\underline{A}}$ such that zero or near zero eigenvalues are enlarged, but large eigenvalues are left essentially unchanged. Following Crosson (1976), the Levenberg-Marquardt algorithm may be derived by minimizing a weighted sum of the residual and solution vectors,

$$
(\underline{\underline{A}} \Delta \underline{m}-\Delta \underline{t})^{T}(\underline{A} \Delta \underline{m}-\Delta \underline{t})+K \Delta \underline{m}^{T} \Delta \underline{m}
$$

which leads to

$$
\Delta \underline{m}=\left(\underline{A}^{T} \underline{\underline{A}}+K \underline{\underline{I}}\right)^{-1} \underline{\underline{A}}^{T} \underline{\Delta t}
$$


The resolution and covariance matrices may be written, respectively, as

$$
\begin{aligned}
& \underline{\underline{R}}=\left(\underline{\underline{A}}^{T} \underline{\underline{A}}+K \underline{\underline{I}}\right)^{-1} \underline{\underline{A}}^{T} \underline{\underline{A}} \\
& \underline{\underline{C}}=\left(\underline{\underline{A}}^{T} \underline{\underline{A}}+K \underline{\underline{I}}\right)^{-1} \underline{\underline{A}}^{T} \underline{\underline{\underline{A}}}\left[\left(\underline{\underline{\underline{A}}}^{T} \underline{\underline{\underline{A}}}\right)+K \underline{\underline{I}}\right]^{-1} .
\end{aligned}
$$

The value of $K$ must be large enough to ensure adequate tapering of the eigenvalues for a stable inversion, but small enough to ensure good resolution and reliable standard errors. When $K$ is less than 1, the resolution and standard error estimates approach those of standard least squares. However, if $K$ is greater than 1 , poor resolution and artificially low standard errors are obtained. In the inversions for this study, the value of $K$ was allowed to vary from a starting value of $10^{-2}$. All final values of $K$ ranged from $10^{-2}$ to $10^{-6}$.

\section{Velocity Model Estimation}

The velocities and crustal structure were estimated using $P$-wave travel times of the four events listed in Table 1. Their preliminary locations were based on an average, regional model of flat, constant velocity layers for southern California developed and used by the U.S. Geological Survey and the California Institute of

TABLE 1

Events Used in the Velocity Model EstimationPreliminary Locations

\begin{tabular}{lccccc}
\hline \multicolumn{1}{c}{ Date } & Origin & Time & Latitude $\left(^{\circ}\right)$ & Longitude $\left(^{\circ}\right)$ & Depth $(\mathrm{km})$ \\
\hline $12 / 13 / 76$ & 0826 & 55.54 & 34.470 & -117.956 & 7.68 \\
$1 / 1 / 77$ & 0100 & 31.73 & 34.458 & -117.964 & 8.58 \\
$3 / 7 / 77$ & 1104 & 35.29 & 34.473 & -117.960 & 8.17 \\
$9 / 6 / 77$ & 0508 & 40.52 & 34.467 & -117.963 & 8.00 \\
\hline
\end{tabular}

Technology. The motivation in determining a different velocity model was to include features of a dipping upper crustal gradient layer and to use local rather than regional velocities in the hypocenter relocations. A local velocity model was necessary because most of the events in the swarm had duration magnitudes of less than 2.0 , and therefore, they were well recorded only at stations having epicentral distances of $60 \mathrm{~km}$ or less. In addition, we believed that a gradient velocity model would be more realistic for an upper crustal model, than constant velocity layers.

The four events used in the velocity model estimation had larger magnitudes than the other events $\left(M_{L}=2.2,2.8,3.0,2.8\right)$ and were well recorded regionally, so there were adequate direct and refracted arrivals to sample shallow and intermediate crustal structure. Unfortunately, the initial locations of the four events are within a $1-\mathrm{km}$ cluster, so it was not possible to have reverse paths in the velocity structure determination. However, the four events had the most reliable preliminary regional locations, and the arrival times used from each event were chosen carefully so as to enhance the azimuthal coverage and distance ranges and to avoid redundant ray paths. The station distribution used for the velocity model estimation is shown in Figure 2. No station corrections to the arrival times were used in the inversion. The preliminary hypocenters of the four events used were fixed in the inversion. The events had been relatively well located using the regional model, and we decided to fix the hypocentral parameters in order to prevent the trade-off between origin 
times, depths, and velocities that would have existed had those parameters been free.

Initial inversions of the travel-time data allowed six free parameters: $v_{0}, k, v_{h}, H_{0}$, $a$, and $b$. The dip parameters, $\alpha$ and $b$, were scaled from units of $\mathrm{km} / \mathrm{deg}$ to $\mathrm{km} / 0.1$ deg so that the partial derivatives of the travel-time function with respect to $a$ and $b$ were of order unity. The other derivatives except that with respect to the gradient, $k$, were of order unity without scaling. The time derivative with respect to the gradient was large, but attempts to scale this derivative to order unity adversely affected the stability of the inversion. The gradient changed little from iteration to iteration, so a number of starting model gradients were tested. An optimum gradient was selected based on the lowest residuals for direct arrivals, and the gradient was fixed at the value in later inversions. By fixing the gradient, the remaining partial derivatives in the matrix $\underline{\underline{A}}$ were of order unity and the conditioning of the problem improved.

The resolution matrix for the inversions was examined. The velocities were well resolved in the sense that the rows of the resolution matrix, or resolving kernels, associated with the model parameters approached the identity matrix. A trade-off existed, however, between the dip parameters, $a$ and $b$, and the depth to the half-

TABLE 2

\begin{tabular}{lcc} 
& \multicolumn{3}{c}{ VELOCITY MODEL } \\
\hline \multicolumn{1}{c}{ Model Parameters } & Starting Model & Final Model \\
\hline Initial layer velocity, $v_{0}$ & $5.00 \mathrm{~km} / \mathrm{sec}$ & $5.70 \pm 0.02 \mathrm{~km} / \mathrm{sec}$ \\
Layer gradient, $k$ & $0.049 \mathrm{sec}^{-1}$ & $0.049 \mathrm{sec}^{-1}$ \\
Half-space velocity & $6.20 \mathrm{~km} / \mathrm{sec}$ & $6.30 \pm 0.05 \mathrm{~km} / \mathrm{sec}$ \\
Depth to half-space at refer- & $15.00 \mathrm{~km}$ & $11.40 \pm 0.86 \mathrm{~km}$ \\
$\quad$ & & \\
$\quad$ ence station, $H_{0}$ & & \\
Latitude dip parameter, $a$ & $0.00 \mathrm{~km} / \mathrm{deg}$ & $11.91 \pm 1.88 \mathrm{~km} / \mathrm{deg}$ \\
Longitude dip parameter, $b$ & $0.00 \mathrm{~km} / \mathrm{deg}$ & $2.30 \pm 0.83 \mathrm{~km} / \mathrm{deg}$ \\
& \\
Reference station LRR: & $34.526^{\circ} \mathrm{N}$ & \\
& $118.028^{\circ} \mathrm{W}$. \\
\hline
\end{tabular}

space at the reference station, $H_{0}$, as would be expected. The final results are listed in Table 2. The layer half-space interface dips approximately $6^{\circ}$ to the northeast. Hadley and Kanamori (1977) found a similar dip for a deeper crustal layer. We stress that this model represents the upper crustal structure only.

\section{Hypocentral Relocations and Discussion}

With the model parameters listed in Table 2, hypocenters of the swarm events were relocated. $S$-wave arrival times were used whenever good readings were available. The $S$-wave velocity model was obtained by using an observed $P$-wave velocity-S-wave velocity ratio, 1.74 , for southern California and applying it to the $P$ wave velocity model obtained in this study. There was an inadequate number of reliable $S$-wave readings from the vertical instruments for any one event to warrant a separate estimation of an $S$-wave velocity model.

More than 150 events $\left(0.5 \leqq M_{L} \leqq 3.0\right)$ were recorded between November 1976 and 1978 in the vicinity of Juniper Hills and Blue Ridge. Their relocated hypocenters are listed in Table 3 and are shown along with the major mapped faults in the area in Figure 4. The stations used in the relocations had hypocentral distances of 2 to $60 \mathrm{~km}$. No station corrections to the arrival times were used in the hypocenter 
TABLE 3

Events Recorded Between November 1976 and 1978 in the Vicinity of the Juniper Hills and Blue Ridge AREas

\begin{tabular}{|c|c|c|c|c|c|c|c|c|c|c|}
\hline Date & $\begin{array}{l}\text { Origin } \\
\text { Time }\end{array}$ & $\begin{array}{l}\text { Latitude } \\
\left(^{\circ}\right)\end{array}$ & $\begin{array}{c}\text { Latitude } \\
\text { Standard } \\
\text { Error }\end{array}$ & $\begin{array}{l}\text { Longitude } \\
\left({ }^{\circ}\right)\end{array}$ & $\begin{array}{c}\text { Longitude } \\
\text { Standard } \\
\text { Error }\end{array}$ & $\begin{array}{c}\text { Depth } \\
(\mathrm{km})\end{array}$ & $\begin{array}{c}\text { Depth } \\
\text { Standard } \\
\text { Error }\end{array}$ & Magnitude & $\begin{array}{c}\text { Sum } \\
\text { Square } \\
\text { Residuals }\end{array}$ & $\begin{array}{c}\text { Root } \\
\text { Mean } \\
\text { Square } \\
\text { Residuals }\end{array}$ \\
\hline $11 / 03 / 76$ & 174100.72 & 34.513 & 0.003 & -117.813 & 0.004 & 6.880 & 0.949 & 2.6 & 0.12 & 0.13 \\
\hline $12 / 05 / 76$ & 121228.09 & 34.473 & 0.007 & -117.950 & 0.008 & 6.655 & 2.331 & 0.6 & 0.18 & 0.19 \\
\hline $12 / 13 / 76$ & 082655.50 & 34.468 & 0.004 & -117.960 & 0.005 & 6.473 & 0.660 & 2.2 & 0.08 & 0.13 \\
\hline $12 / 13 / 76$ & 091354.64 & 34.472 & 0.010 & -117.963 & 0.010 & 7.544 & 1.275 & 1.5 & 0.07 & 0.15 \\
\hline $12 / 13 / 76$ & 153752.84 & 34.473 & 0.003 & -117.958 & 0.005 & 8.272 & 1.057 & 1.5 & 0.11 & 0.14 \\
\hline $12 / 13 / 76$ & 154523.75 & 34.473 & 0.002 & -117.957 & 0.004 & 6.602 & 0.583 & 2.1 & 0.10 & 0.11 \\
\hline $12 / 13 / 76$ & 160126.25 & 34.473 & 0.004 & -117.956 & 0.004 & 8.889 & 0.943 & 0.7 & 0.06 & 0.11 \\
\hline $12 / 13 / 76$ & 194311.65 & 34.474 & 0.003 & -117.959 & 0.004 & 6.708 & 0.926 & 0.7 & 0.05 & 0.11 \\
\hline $12 / 15 / 76$ & 184942.12 & 34.472 & 0.002 & -117.949 & 0.004 & 6.551 & 0.563 & 1.4 & 0.05 & 0.09 \\
\hline $12 / 16 / 76$ & 015316.12 & 34.472 & 0.004 & -117.951 & 0.007 & 7.472 & 0.713 & 0.9 & 0.15 & 0.15 \\
\hline $12 / 16 / 76$ & 015704.40 & 34.382 & 0.028 & -118.033 & 0.032 & 9.542 & 2.705 & 0.7 & 0.02 & 0.13 \\
\hline $12 / 17 / 76$ & 041042.77 & 34.467 & 0.008 & -117.960 & 0.008 & 8.821 & 1.800 & 1.2 & 0.18 & 0.17 \\
\hline $12 / 18 / 76$ & 205506.53 & 34.475 & 0.06 & -117.956 & 0.006 & 10.231 & 1.568 & 0.7 & 0.07 & 0.13 \\
\hline $12 / 18 / 76$ & 210039.47 & 34.477 & 0.005 & -117.954 & 0.006 & 8.968 & 1.509 & 0.6 & 0.10 & 0.14 \\
\hline $12 / 19 / 76$ & 143604.09 & 34.472 & 0.004 & -117.960 & 0.005 & 7.740 & 1.359 & 0.8 & 0.07 & 0.12 \\
\hline $12 / 20 / 76$ & 040322.28 & 34.482 & 0.002 & -117.959 & 0.002 & 10.184 & 0.630 & 0.6 & 0.00 & 0.05 \\
\hline $12 / 21 / 76$ & 193431.58 & 34.475 & 0.004 & $-\mathbf{I 1 7 . 9 5 5}$ & 0.006 & 5.739 & 1.123 & 0.6 & 0.05 & 0.12 \\
\hline $12 / 21 / 76$ & 212032.07 & 34.493 & 0.005 & -117.946 & 0.007 & 12.717 & 1.500 & 0.7 & 0.04 & 0.14 \\
\hline $12 / 22 / 76$ & 190347.40 & 34.473 & 0.003 & -117.952 & 0.004 & 5.620 & 0.840 & 0.5 & 0.02 & 0.08 \\
\hline $12 / 23 / 76$ & 081004.75 & 34.475 & 0.005 & -117.955 & 0.007 & 7.917 & 1.402 & 0.8 & 0.19 & 0.18 \\
\hline $12 / 26 / 76$ & 121601.92 & 34.472 & 0.004 & -117.958 & 0.005 & 6.955 & 1.080 & 0.7 & 0.10 & 0.13 \\
\hline $12 / 29 / 76$ & 193906.78 & 34.469 & 0.003 & -117.963 & 0.004 & 7.812 & 0.905 & 0.8 & 0.04 & 0.10 \\
\hline $12 / 30 / 76$ & 203156.11 & 34.468 & 0.005 & -118.000 & 0.008 & 7.764 & 1.194 & 0.7 & 0.07 & 0.13 \\
\hline $12 / 31 / 76$ & 045120.84 & 34.467 & 0.004 & -118.012 & 0.008 & 7.598 & 1.174 & 0.9 & 0.06 & 0.12 \\
\hline $12 / 31 / 76$ & 184324.39 & 34.468 & 0.003 & -117.951 & 0.005 & 7.472 & 0.941 & 1.1 & 0.06 & 0.11 \\
\hline $12 / 31 / 76$ & 184346.28 & 34.468 & 0.003 & -117.950 & 0.006 & 8.117 & 1.036 & 1.2 & 0.06 & 0.11 \\
\hline $01 / 01 / 77$ & 010031.76 & 34.464 & 0.005 & -117.960 & 0.006 & 6.784 & 0.905 & 2.8 & 0.06 & 0.11 \\
\hline $01 / 01 / 77$ & 013507.66 & 34.476 & 0.006 & -117.967 & 0.007 & 8.436 & 1.775 & 1.2 & 0.17 & 0.19 \\
\hline $01 / 01 / 77$ & 015743.51 & 34.470 & 0.01 & -117.948 & 0.002 & 6.335 & 0.283 & 1.1 & 0.03 & 0.03 \\
\hline $01 / 01 / 77$ & 043839.58 & 34.471 & 0.004 & -117.955 & 0.006 & 7.325 & 1.240 & 0.8 & 0.10 & 0.14 \\
\hline $01 / 01 / 77$ & 222524.45 & 34.473 & 0.004 & -117.960 & 0.005 & 8.108 & 1.097 & 1.3 & 0.08 & 0.13 \\
\hline $01 / 02 / 77$ & 023004.14 & 34.470 & 0.04 & -117.958 & 0.005 & 6.544 & 0.685 & 0.7 & 0.08 & 0.14 \\
\hline $01 / 12 / 77$ & 052542.82 & 34.473 & 0.04 & -117.955 & 0.005 & 6.087 & 0.658 & 1.2 & 0.05 & 0.11 \\
\hline $01 / 14 / 77$ & 010146.40 & 34.468 & 0.004 & -117.954 & 0.006 & 8.966 & 1.474 & 1.9 & 0.11 & 0.17 \\
\hline $01 / 15 / 77$ & 051053.12 & 34.471 & 0.03 & -117.957 & 0.003 & 8.298 & 0.800 & 0.6 & 0.03 & 0.08 \\
\hline $01 / 15 / 77$ & 053229.59 & 34.472 & 0.003 & -117.957 & 0.004 & 6.968 & 0.868 & 0.6 & 0.05 & 0.10 \\
\hline $01 / 15 / 77$ & 053305.90 & 34.469 & 0.004 & -117.959 & 0.004 & 6.990 & 1.074 & 0.8 & 0.08 & 0.13 \\
\hline $01 / 15 / 77$ & 060242.92 & 34.466 & 0.002 & -117.952 & 0.002 & 7.044 & 0.490 & 0.8 & 0.01 & 0.05 \\
\hline $01 / 15 / 77$ & 082242.14 & 34.470 & 0.002 & -117.960 & 0.003 & 8.103 & 0.704 & 0.5 & 0.02 & 0.08 \\
\hline $01 / 19 / 77$ & 130748.04 & 34.491 & 0.004 & -117.979 & 0.007 & 9.225 & 1.276 & 1.3 & 0.09 & 0.15 \\
\hline $01 / 22 / 77$ & 050214.46 & 34.486 & 0.007 & -117.961 & 0.008 & 12.036 & 1.723 & 0.4 & 0.12 & 0.18 \\
\hline $01 / 24 / 77$ & 190841.75 & 34.454 & 0.04 & -117.937 & 0.006 & 9.630 & 0.782 & 1.2 & 0.08 & 0.14 \\
\hline $01 / 24 / 77$ & 214645.93 & 34.472 & 0.005 & -117.950 & 0.006 & 8.682 & 1.319 & 0.7 & 0.10 & 0.16 \\
\hline $01 / 24 / 77$ & 215915.90 & 34.441 & 0.003 & -117.946 & 0.004 & 9.504 & 0.400 & 1.5 & 0.06 & 0.10 \\
\hline $01 / 26 / 77$ & 074333.21 & 34.446 & 0.004 & -117.943 & 0.006 & 9.299 & 1.095 & 1.6 & 0.18 & 0.17 \\
\hline $01 / 29 / 77$ & 051604.05 & 34.477 & 0.005 & -117.959 & 0.007 & 7.161 & 1.783 & 0.7 & 0.09 & 0.15 \\
\hline $01 / 29 / 77$ & 055942.43 & 34.475 & 0.002 & -117.951 & 0.002 & 5.955 & 0.359 & 0.8 & 0.07 & 0.05 \\
\hline $01 / 29 / 77$ & 072456.38 & 34.442 & 0.003 & -117.709 & 0.004 & 2.974 & 0.782 & 1.5 & 0.01 & 0.08 \\
\hline $02 / 12 / 77$ & 113651.75 & 34.450 & 0.003 & -117.924 & 0.003 & 9.593 & 0.519 & 1.5 & 0.05 & 0.09 \\
\hline $02 / 12 / 77$ & 215705.92 & 34.479 & 0.008 & -117.965 & 0.007 & 9.921 & 1.884 & 1.4 & 0.23 & 0.21 \\
\hline $02 / 13 / 77$ & 210627.98 & 34.476 & 0.02 & -117.955 & 0.003 & 8.010 & 0.826 & 1.8 & 0.03 & 0.07 \\
\hline $02 / 14 / 77$ & 091650.53 & 34.482 & 0.002 & -117.864 & 0.002 & 4.282 & 0.491 & 2.0 & 0.06 & 0.08 \\
\hline $02 / 21 / 77$ & 164705.59 & 34.445 & 0.003 & -117.942 & 0.003 & 11.240 & 0.402 & 0.8 & 0.01 & 0.06 \\
\hline $02 / 22 / 77$ & 150122.60 & 34.472 & 0.002 & -117.952 & 0.002 & 7.277 & 0.672 & 0.8 & 0.03 & 0.08 \\
\hline $02 / 24 / 77$ & 222421.71 & 34.376 & 0.005 & -117.665 & 0.008 & 8.666 & 0.578 & 0.8 & 0.03 & 0.10 \\
\hline $02 / 25 / 77$ & 190155.70 & 34.452 & 0.006 & -117.917 & 0.006 & 7.810 & 1.001 & 1.7 & 0.24 & 0.19 \\
\hline $02 / 26 / 77$ & 170532.63 & 34.474 & 0.004 & -117.954 & 0.003 & 7.792 & 0.797 & 1.3 & 0.04 & 0.09 \\
\hline $02 / 27 / 77$ & 073109.72 & 34.468 & 0.003 & -117.961 & 0.002 & 7.785 & 0.610 & 0.7 & 0.04 & 0.08 \\
\hline $02 / 27 / 77$ & 104123.60 & 34.477 & 0.002 & -117.959 & 0.003 & 7.575 & 0.863 & 1.7 & 0.07 & 0.10 \\
\hline $02 / 27 / 77$ & 144837.60 & 34.476 & 0.004 & -117.962 & 0.005 & 7.494 & 0.921 & 2.2 & 0.22 & 0.17 \\
\hline $03 / 01 / 77$ & 111718.09 & 34.473 & 0.02 & -117.951 & 0.002 & 6.658 & 0.462 & 0.6 & 0.01 & 0.05 \\
\hline $03 / 05 / 77$ & 144311.69 & 34.468 & 0.002 & -117.958 & 0.004 & 1.039 & 1.040 & 2.1 & 0.09 & 0.11 \\
\hline $03 / 06 / 77$ & 130552.59 & 34.479 & 0.003 & -117.948 & 0.003 & 9.505 & 0.753 & 1.0 & 0.06 & 0.10 \\
\hline
\end{tabular}


TABLE 3-Continued

\begin{tabular}{|c|c|c|c|c|c|c|c|c|c|c|}
\hline Date & $\begin{array}{l}\text { Origin } \\
\text { Time }\end{array}$ & $\begin{array}{c}\text { Latitude } \\
\left({ }^{\circ}\right)\end{array}$ & $\begin{array}{c}\text { Latitude } \\
\text { Standard } \\
\text { Error }\end{array}$ & $\begin{array}{l}\text { Longitude } \\
\left({ }^{\circ}\right)\end{array}$ & $\begin{array}{l}\text { Longitude } \\
\text { Standard } \\
\text { Error }\end{array}$ & $\begin{array}{c}\text { Depth } \\
(\mathrm{km})\end{array}$ & $\begin{array}{l}\text { Depth } \\
\text { Standard } \\
\text { Error }\end{array}$ & Magnitude & $\begin{array}{c}\text { Sum } \\
\text { Square } \\
\text { Residuals }\end{array}$ & $\begin{array}{c}\text { Root } \\
\text { Mean } \\
\text { Square } \\
\text { Residuals }\end{array}$ \\
\hline $03 / 07 / 77$ & 093612.90 & 34.469 & 0.005 & -117.946 & 0.006 & 4.457 & 1.497 & 2.4 & 0.37 & 0.21 \\
\hline $03 / 07 / 77$ & 110435.23 & 34.469 & 0.004 & -117.959 & 0.006 & 6.496 & 0.987 & 3.0 & 0.16 & 0.15 \\
\hline $03 / 08 / 77$ & 041414.29 & 34.467 & 0.005 & -117.961 & 0.006 & 9.788 & 1.509 & 1.7 & 0.02 & 0.10 \\
\hline $03 / 12 / 77$ & 202458.24 & 34.468 & 0.003 & -117.961 & 0.006 & 6.855 & 1.347 & 2.2 & 0.20 & 0.17 \\
\hline $03 / 21 / 77$ & 012853.86 & 34.473 & 0.003 & -117.961 & 0.004 & 7.274 & 0.767 & 1.7 & 0.06 & 0.11 \\
\hline $03 / 23 / 77$ & 051515.24 & 34.468 & 0.002 & -117.962 & 0.004 & 7.198 & 0.822 & 2.5 & 0.13 & 0.13 \\
\hline $03 / 24 / 77$ & 045711.90 & 34.456 & 0.010 & -117.962 & 0.007 & 8.595 & 1.537 & 0.8 & 0.10 & 0.16 \\
\hline $03 / 26 / 77$ & 065832.03 & 34.466 & 0.002 & -117.964 & 0.003 & 10.431 & 0.647 & 1.6 & 0.09 & 0.10 \\
\hline $03 / 26 / 77$ & 151655.52 & 34.455 & 0.010 & -117.967 & 0.007 & 8.257 & 1.533 & 0.3 & 0.07 & 0.16 \\
\hline $03 / 27 / 77$ & 175614.84 & 34.467 & 0.005 & -117.964 & 0.003 & 7.345 & 0.795 & 0.7 & 0.02 & 0.08 \\
\hline $03 / 30 / 77$ & 130842.58 & 34.489 & 0.003 & -118.059 & 0.005 & 10.503 & 1.054 & 2.0 & 0.10 & 0.14 \\
\hline $04 / 05 / 77$ & 044344.68 & 34.459 & 0.001 & -117.965 & 0.001 & 7.971 & 0.353 & 0.6 & 0.01 & 0.04 \\
\hline $04 / 05 / 77$ & 104230.72 & 34.475 & 0.02 & -117.945 & 0.002 & 6.875 & 0.395 & 1.6 & 0.01 & 0.04 \\
\hline $04 / 07 / 77$ & 194753.07 & 34.469 & 0.04 & -117.959 & 0.003 & 8.463 & 1.628 & 1.4 & 0.01 & 0.08 \\
\hline $04 / 09 / 77$ & 172130.04 & 34.469 & 0.004 & -117.967 & 0.005 & 8.522 & 2.073 & 1.3 & 0.04 & 0.12 \\
\hline $04 / 10 / 77$ & 072602.66 & 34.457 & 0.003 & -117.948 & 0.003 & 8.148 & 0.864 & 0.5 & 0.04 & 0.09 \\
\hline $04 / 11 / 77$ & 140502.93 & 34.476 & 0.02 & -117.963 & 0.003 & 7.735 & 0.666 & 1.1 & 0.02 & 0.07 \\
\hline $04 / 12 / 77$ & 161417.76 & 34.446 & 0.03 & -117.960 & 0.003 & 13.616 & 0.805 & 0.3 & 0.05 & 0.07 \\
\hline $04 / 13 / 77$ & 105750.60 & 34.471 & 0.003 & -117.955 & 0.005 & 9.208 & 1.436 & 2.0 & 0.20 & 0.16 \\
\hline $04 / 13 / 77$ & 140658.42 & 34.465 & 0.002 & -117.954 & 0.002 & 8.338 & 0.513 & 0.4 & 0.02 & 0.06 \\
\hline $04 / 14 / 77$ & 074129.69 & 34.471 & 0.03 & -117.958 & 0.003 & 7.565 & 0.958 & 1.4 & 0.04 & 0.09 \\
\hline $04 / 28 / 77$ & 063802.58 & 34.469 & 0.02 & -117.960 & 0.002 & 8.167 & 0.547 & 1.4 & 0.01 & 0.05 \\
\hline $04 / 28 / 77$ & 190806.73 & 34.480 & 0.001 & -117.951 & 0.000 & 5.451 & 0.100 & 0.4 & 0.00 & 0.01 \\
\hline $05 / 02 / 77$ & 063119.72 & 34.463 & 0.002 & -117.958 & 0.002 & 7.566 & 0.372 & 0.8 & 0.05 & 0.08 \\
\hline $05 / 05 / 77$ & 095547.88 & 34.472 & 0.01 & -117.959 & 0.002 & 8.088 & 0.192 & 1.8 & 0.00 & 0.01 \\
\hline $06 / 21 / 77$ & 120316.19 & 34.468 & 0.003 & -117.961 & 0.004 & 7.773 & 1.975 & 1.9 & 0.13 & 0.14 \\
\hline $07 / 25 / 77$ & 211925.19 & 34.460 & 0.002 & -117.916 & 0.003 & 10.292 & 0.916 & 1.7 & 0.03 & 0.09 \\
\hline $07 / 27 / 77$ & 012433.78 & 34.427 & 0.006 & -117.721 & 0.007 & 5.443 & 1.680 & 1.5 & 0.34 & 0.26 \\
\hline $07 / 27 / 77$ & 0133.47 .52 & 34.431 & 0.03 & -117.715 & 0.004 & 5.697 & 0.940 & 1.6 & 0.27 & 0.16 \\
\hline $07 / 27 / 77$ & 014409.04 & 34.424 & 0.05 & -117.718 & 0.005 & 4.563 & 0.800 & 1.3 & 0.07 & 0.13 \\
\hline $07 / 27 / 77$ & 021442.26 & 34.428 & 0.006 & -117.732 & 0.016 & 3.760 & 2.416 & 0.6 & 0.13 & 0.18 \\
\hline $07 / 27 / 77$ & 021526.04 & 34.435 & 0.005 & -117.688 & 0.014 & 6.263 & 1.671 & 0.7 & 0.18 & 0.18 \\
\hline $07 / 27 / 77$ & 022128.81 & 34.432 & 0.010 & -117.731 & 0.023 & 3.936 & 2.472 & 0.6 & 0.11 & 0.24 \\
\hline $07 / 27 / 77$ & 023360.53 & 34.432 & $0.0-3$ & -117.724 & 0.005 & 3.888 & 0.909 & 1.9 & 0.08 & 0.12 \\
\hline $07 / 27 / 77$ & 024159.41 & 34.429 & 0.006 & -117.665 & 0.016 & 7.966 & 2.107 & 0.7 & 0.08 & 0.17 \\
\hline $07 / 27 / 77$ & 031716.70 & 34.421 & 0.011 & -117.703 & 0.023 & 5.975 & 3.661 & 0.6 & 0.41 & 0.32 \\
\hline $07 / 27 / 77$ & 053023.92 & 34.435 & 0.006 & -117.712 & 0.008 & 4.085 & 1.344 & 1.0 & 0.28 & 0.20 \\
\hline $07 / 27 / 77$ & 053054.93 & 34.428 & 0.005 & -117.709 & 0.006 & 4.109 & 1.276 & 0.8 & 0.21 & 0.17 \\
\hline $07 / 27 / 77$ & 055719.30 & 34.420 & 0.005 & -117.720 & 0.005 & 4.422 & 1.410 & 1.6 & 0.25 & 0.21 \\
\hline $07 / 27 / 77$ & 061751.18 & 34.424 & 0.009 & -117.707 & 0.008 & 4.501 & 1.867 & 0.6 & 0.30 & 0.24 \\
\hline $07 / 27 / 77$ & 062239.16 & 34.428 & 0.005 & -117.725 & 0.004 & 4.424 & 0.958 & 0.9 & 0.14 & 0.14 \\
\hline $07 / 27 / 77$ & 082626.04 & 34.432 & 0.004 & -117.721 & 0.004 & 3.975 & 0.916 & 2.0 & 0.14 & 0.13 \\
\hline $08 / 05 / 77$ & 105345.45 & 34.505 & 0.003 & -118.091 & 0.004 & 10.552 & 1.048 & 1.5 & 0.07 & 0.12 \\
\hline $08 / 06 / 77$ & 080327.80 & 34.502 & 0.005 & -118.111 & 0.006 & 8.880 & 1.220 & 0.7 & 0.17 & 0.15 \\
\hline $08 / 21 / 77$ & 051908.72 & 34.532 & 0.006 & -118.099 & 0.007 & 11.457 & 0.977 & 0.7 & 0.14 & 0.17 \\
\hline $08 / 24 / 77$ & 223506.19 & 34.459 & 0.003 & -117.960 & 0.004 & 7.502 & 0.894 & 1.3 & 0.03 & 0.10 \\
\hline $08 / 28 / 77$ & 110957.62 & 34.463 & 0.004 & -117.956 & 0.003 & 8.021 & 1.507 & 0.7 & 0.06 & 0.11 \\
\hline $09 / 01 / 77$ & 141852.70 & 34.472 & 0.002 & -117.938 & 0.003 & 6.498 & 0.473 & 1.7 & 0.01 & 0.06 \\
\hline $09 / 06 / 77$ & 050840.45 & 34.467 & 0.003 & -117.969 & 0.004 & 6.806 & 0.666 & 2.8 & 0.15 & 0.13 \\
\hline $09 / 06 / 77$ & I03211.79 & 34.462 & 0.003 & -117.970 & 0.003 & 8.312 & 0.656 & 0.6 & 0.06 & 0.11 \\
\hline $09 / 13 / 77$ & 112638.81 & 34.395 & 0.003 & -117.676 & 0.003 & 9.088 & 1.003 & 1.5 & 0.13 & 0.12 \\
\hline $09 / 13 / 77$ & 235604.32 & 34.372 & 0.02 & -117.676 & 0.003 & 7.811 & 0.918 & 1.4 & 0.06 & 0.10 \\
\hline $09 / 14 / 77$ & 184528.68 & 34.452 & 0.004 & -117.961 & 0.005 & 8.395 & 1.144 & 0.6 & 0.10 & 0.14 \\
\hline $09 / 21 / 77$ & 143739,00 & 34.463 & 0.003 & -117.967 & 0.004 & 8.456 & 0.715 & 0.8 & 0.08 & 0.10 \\
\hline $09 / 27 / 77$ & 220942.00 & 34.397 & 0.002 & -117.669 & 0.003 & 9.221 & 0.766 & 1.5 & 0.02 & 0.07 \\
\hline $09 / 28 / 77$ & 010752.24 & 34.469 & 0.003 & -117.967 & 0.008 & 8.272 & 1.221 & 1.8 & 0.11 & 0.14 \\
\hline $10 / 03 / 77$ & 133827.76 & 34.404 & 0.06 & -117.692 & 0.008 & 4.848 & 1.210 & 1.6 & 0.09 & 0.15 \\
\hline $10 / 12 / 77$ & 174421.29 & 34.475 & 0.004 & -117.986 & 0.004 & 8.920 & 1.065 & 0.6 & 0.06 & 0.11 \\
\hline $10 / 12 / 77$ & 192349.82 & 34.488 & 0.003 & -118.033 & 0.004 & 11.221 & 0.970 & 0.6 & 0.01 & 0.06 \\
\hline $10 / 23 / 77$ & 092503.40 & 34.457 & 0.03 & -117.970 & 0.003 & 8.068 & 0.659 & 1.6 & 0.10 & 0.11 \\
\hline $10 / 25 / 77$ & 134839.52 & 34.480 & 0.03 & -117.938 & 0.004 & 5.545 & 1.387 & 1.8 & 0.04 & 0.10 \\
\hline $11 / 04 / 77$ & 083355.32 & 34.461 & 0.004 & -117.887 & 0.003 & 4.681 & 0.661 & 0.6 & 0.05 & 0.10 \\
\hline $11 / 23 / 77$ & 215718.69 & 34.477 & 0.009 & -118.190 & 0.005 & 7.871 & 1.552 & 1.4 & 0.22 & 0.19 \\
\hline $12 / 01 / 77$ & 222317.24 & 34.457 & $0.0-4$ & -118.175 & 0.008 & 6.691 & 0.921 & 1.4 & 0.13 & 0.14 \\
\hline $12 / 29 / 77$ & 014150.58 & 34.432 & 0.004 & -117.949 & 0.006 & 8.171 & 0.858 & 0.7 & 0.01 & 0.09 \\
\hline $01 / 12 / 78$ & 215530.93 & 34.522 & 0.003 & -117.943 & 0.005 & 6.868 & 0.705 & 0.6 & 0.02 & 0.09 \\
\hline $01 / 26 / 78$ & 200856.18 & 34.473 & 0.002 & -117.988 & 0.003 & 7.981 & 0.731 & 1.6 & 0.08 & 0.11 \\
\hline $01 / 31 / 78$ & 121930.42 & 34.458 & 0.05 & -1117.919 & 0.005 & 7.018 & 1.567 & 1.0 & 0.19 & 0.18 \\
\hline
\end{tabular}


EARTHQUAKE SWARM EVENTS NEAR PALMDALE, CALIFORNIA

TABLE 3-Continued

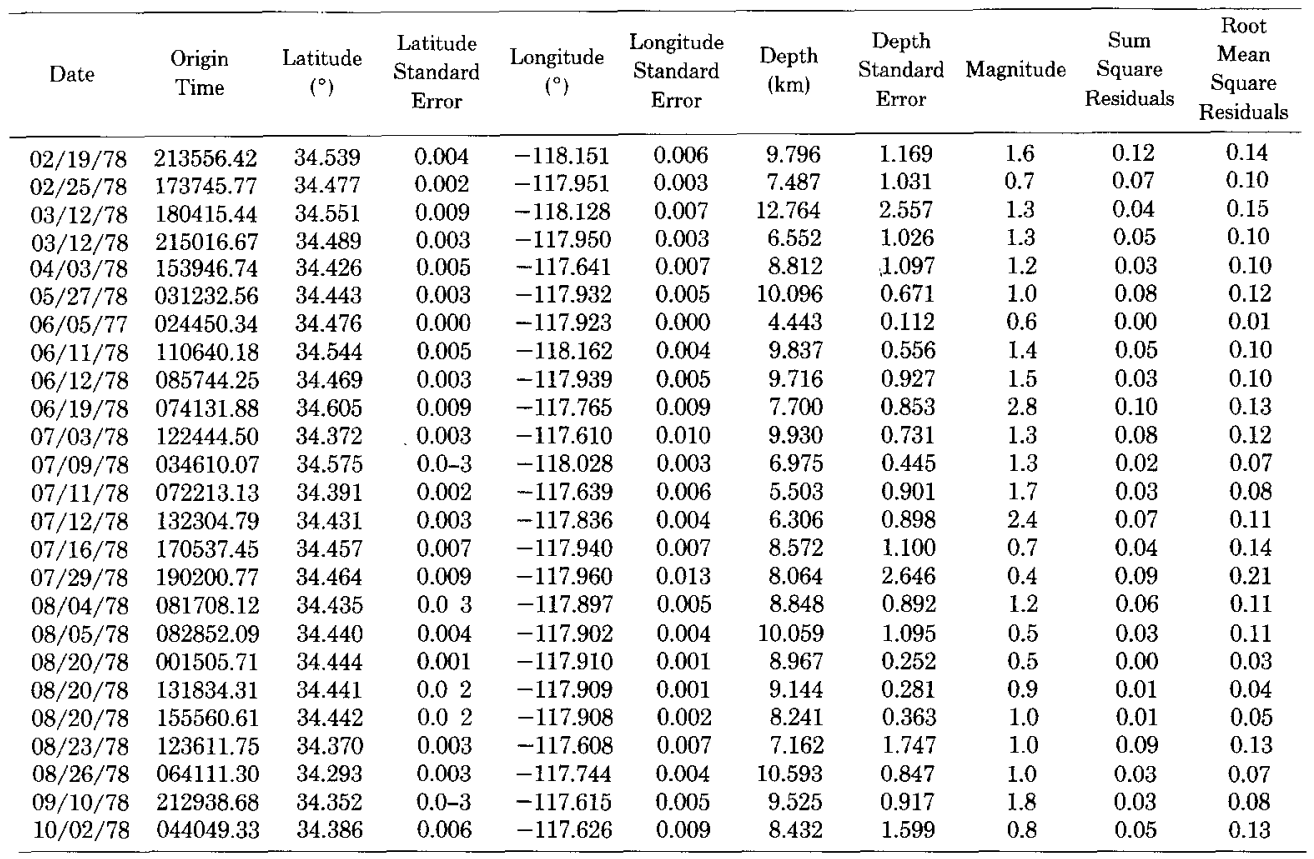

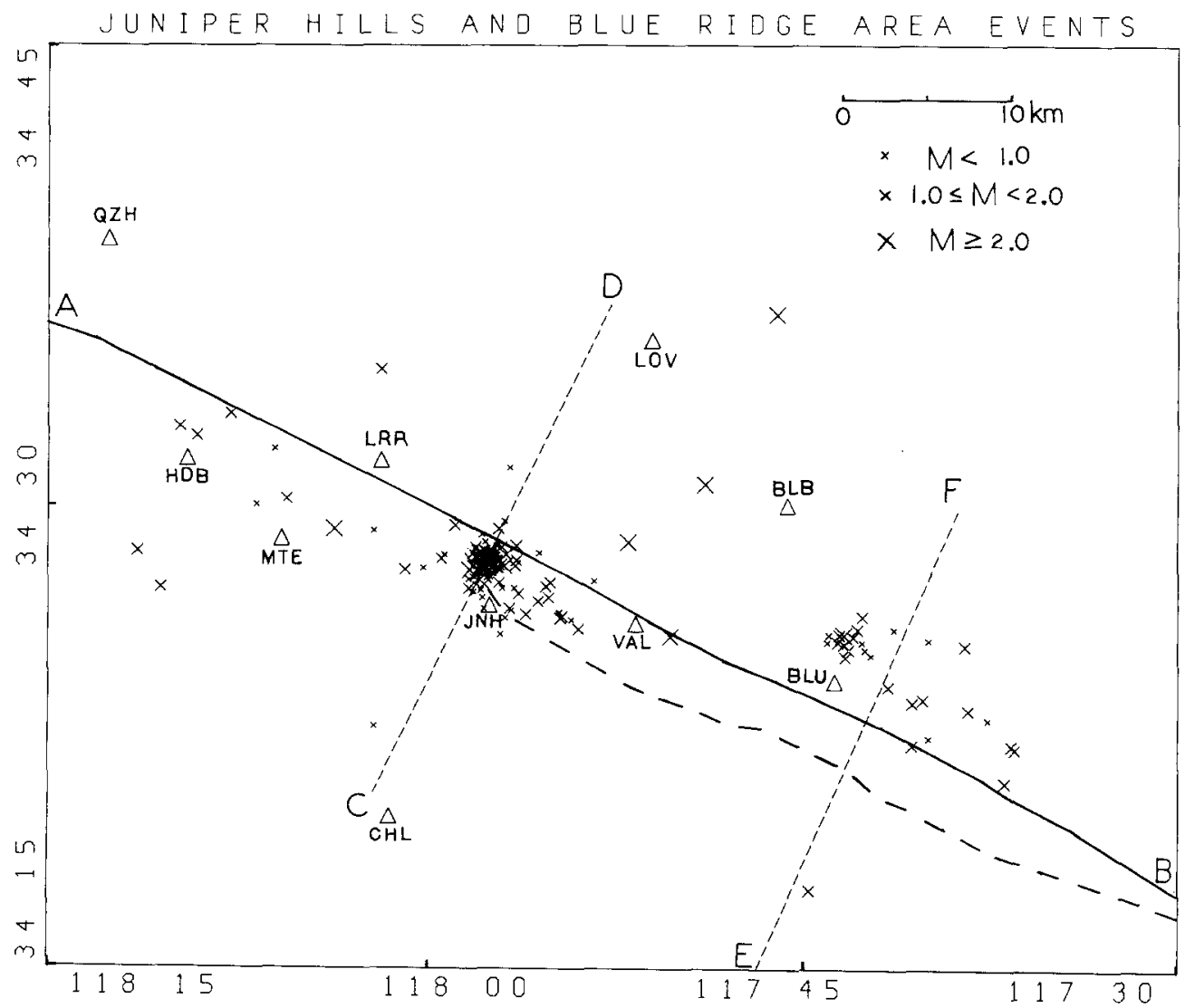

Fig. 4. Final epicenters shown relative to the San Andreas Fault (solid) and the Punchbowl Fault (dashed). Triangles represent stations. Letters refer to the cross sections shown in Figures 6 through 9. 
relocation. Examination of all residuals after the events were relocated indicated a zero mean residual for each station, and thus, no station corrections were warranted. The rms residuals for the refined hypocenters were all less than 0.2 sec and were typically less than $0.1 \mathrm{sec}$.

Most of the events are clustered between the mapped traces of the San Andreas and Punchbowl faults in a region containing numerous mapped short fault segments

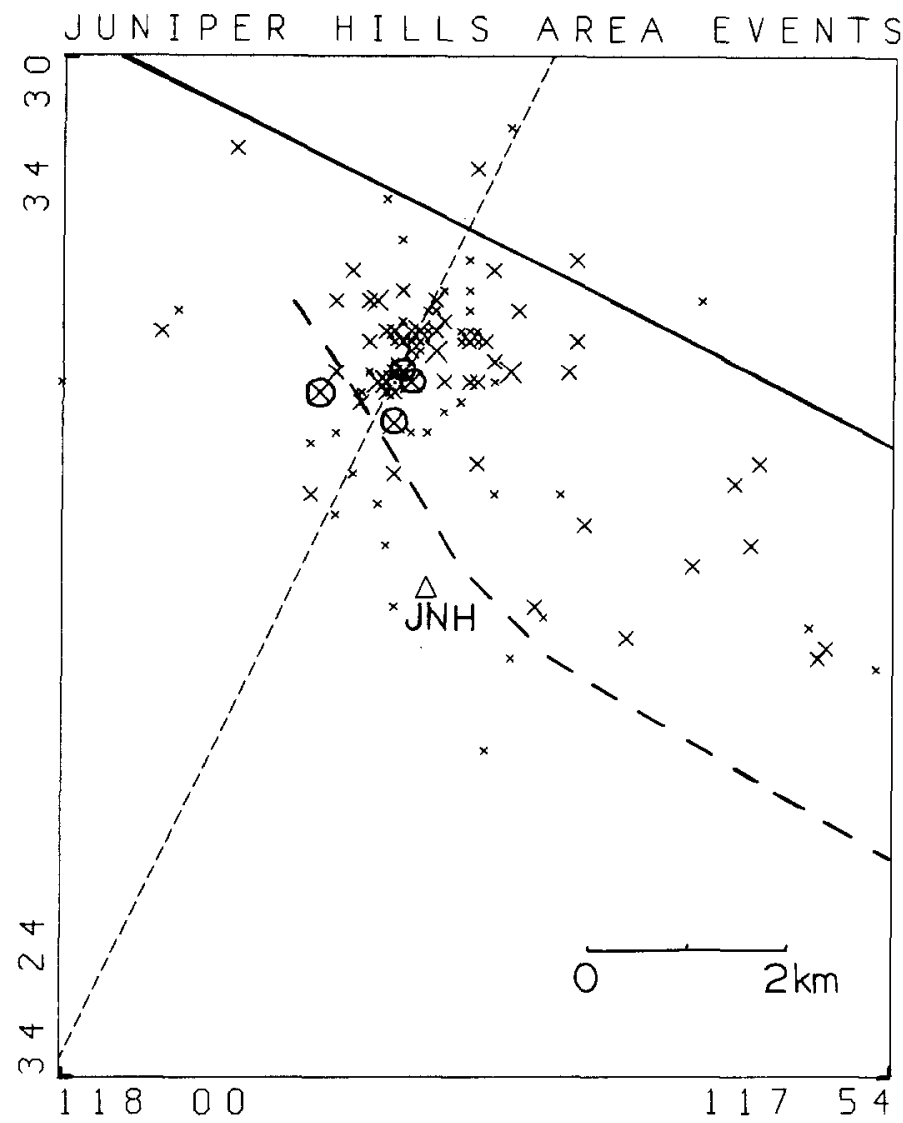

FIg. 5. Close-up map view of the activity near Juniper Hills. The events used in the velocity model estimation are circled.

having the same northwest-southeast orientation as the San Andreas Fault. Uncertainties in the epicenters range from less than $0.5 \mathrm{~km}$ for well-recorded events to 1.0 $\mathrm{km}$ for smaller events. A second cluster of activity is near Blue Ridge. In this area, epicenters exhibit greater uncertainty, approximately $1.0 \mathrm{~km}$.

Figure 5 shows a close-up of the activity at Juniper Hills. The four events used in the velocity model estimation are shown relative to the other swarm events and the major mapped fault traces.

All events are projected onto the San Andreas Fault in cross section A-B (Figure 4), as shown in Figure 6. The earthquakes range in depth from 3.0 to $12.0 \mathrm{~km}$ with uncertainties of less than $1 \mathrm{~km}$ for the best locations to about $2.5 \mathrm{~km}$ for the smallest events. The depth uncertainties were typically less than $1.5 \mathrm{~km}$.

Swarm events within $10 \mathrm{~km}$ are projected along lines perpendicular to the San Andreas Fault in cross sections C-D and E-F (Figure 4), as shown in Figures 7 and 8. Some scatter is apparent, but the general trend is vertical.

The most interesting feature of the swarm is an apparent migration of activity 


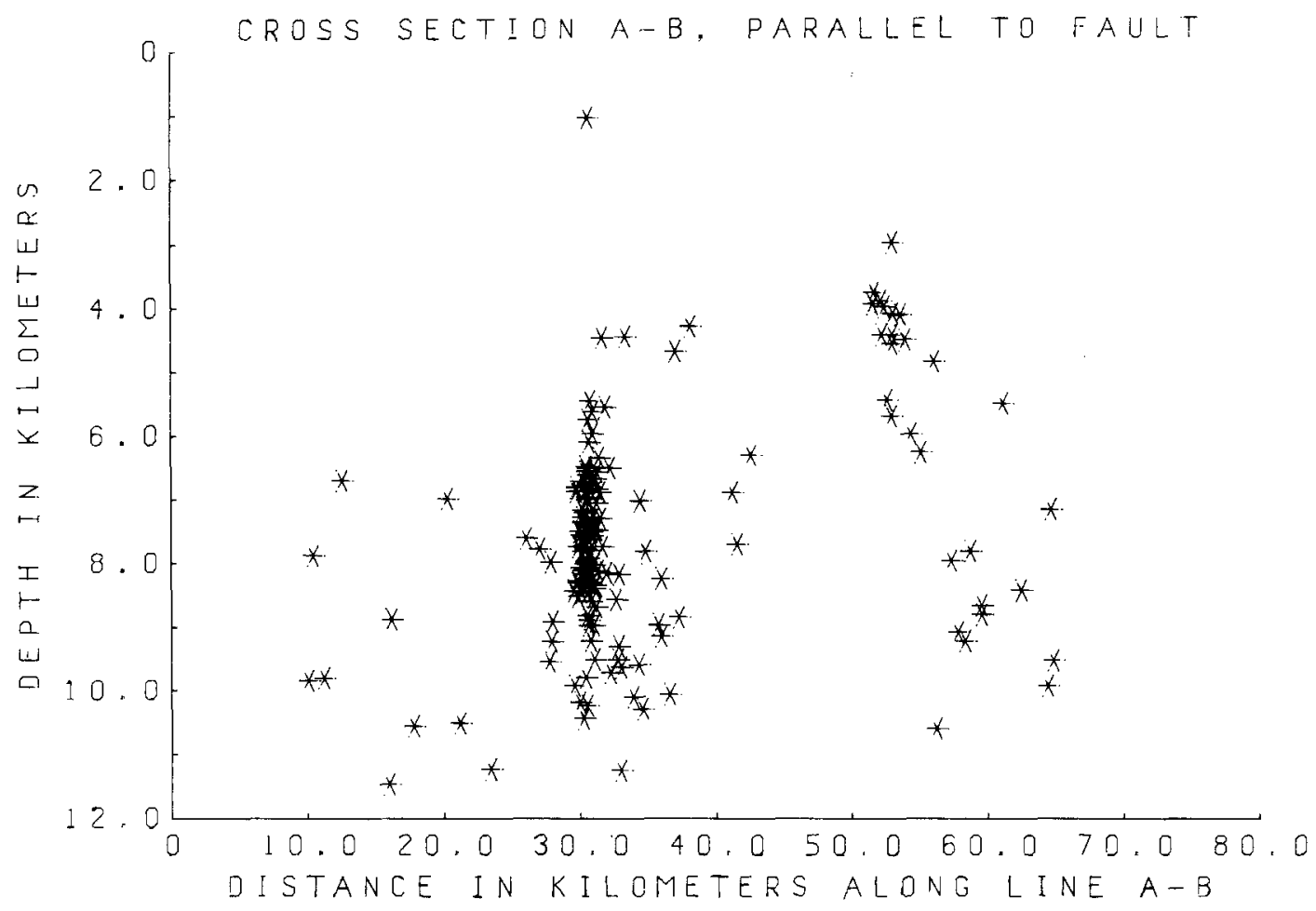

Fig. 6. Cross section taken parallel to the San Andreas Fault showing the depth distribution of the events.

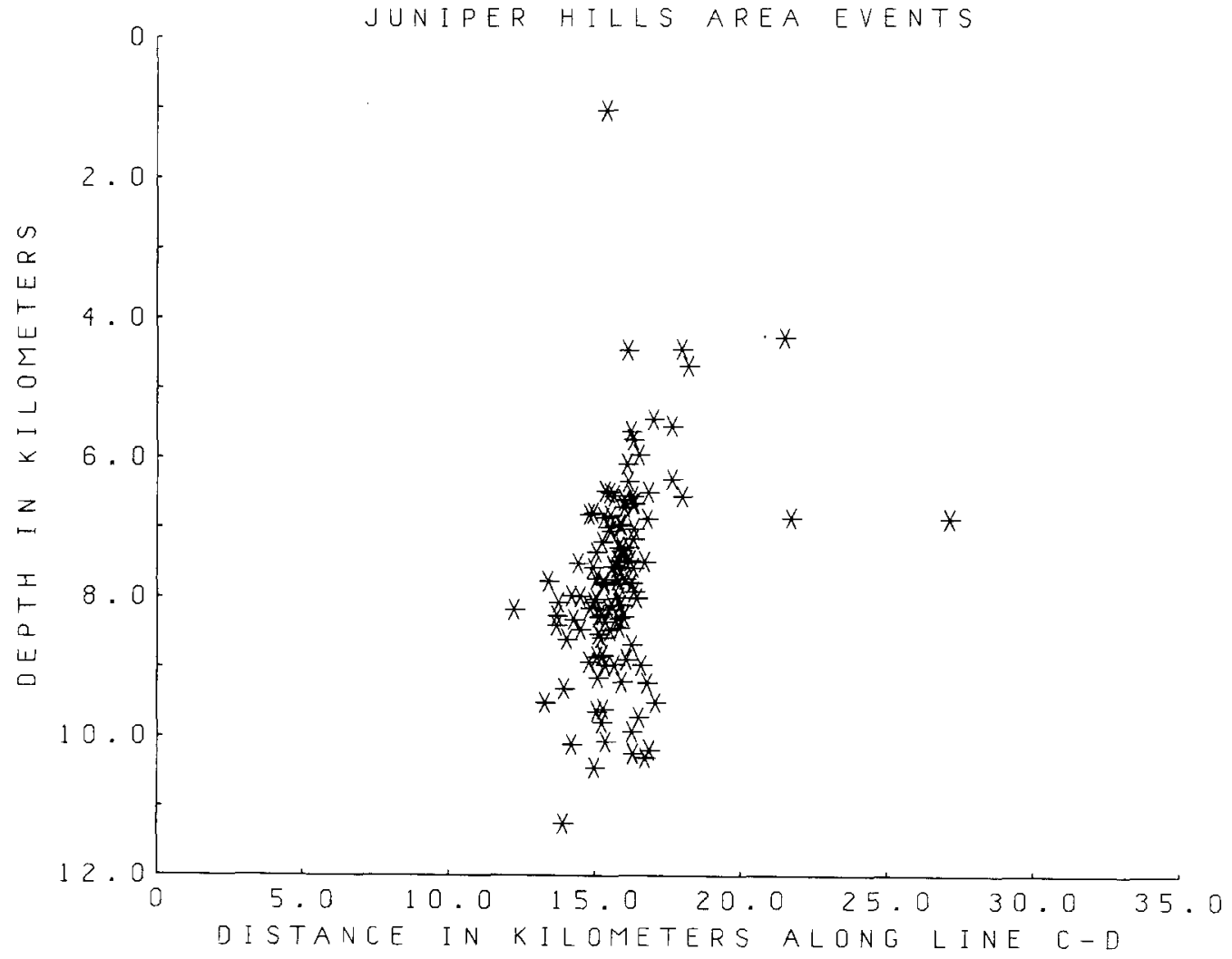

FIG. 7. Cross section taken normal to the San Andreas Fault showing the depth distribution of events in the vicinity of Juniper Hills. 
with time along the San Andreas Fault. The time distribution of the earthquakes projected onto the San Andreas (line A-B, Figure 4) is shown in Figure 9. The Juniper Hills cluster of activity in the early months of the swarm is evident, but a migration with time from this cluster outward along the San Andreas Fault is apparent in the later months of the swarm. The Juniper Hills activity continued in intervals during this time. The initial cluster, which was approximately $5 \mathrm{~km}$ in diameter, has expanded to fill a $60-\mathrm{km}$ segment of the San Andreas Fault. The apparent migration rate is approximately $15 \mathrm{~km} / \mathrm{yr}$. Johnson and Hadley (1976) observed a migration rate of $0.5 \mathrm{~km} / \mathrm{hr}$ for the Brawley swarm sequence of 1975 .

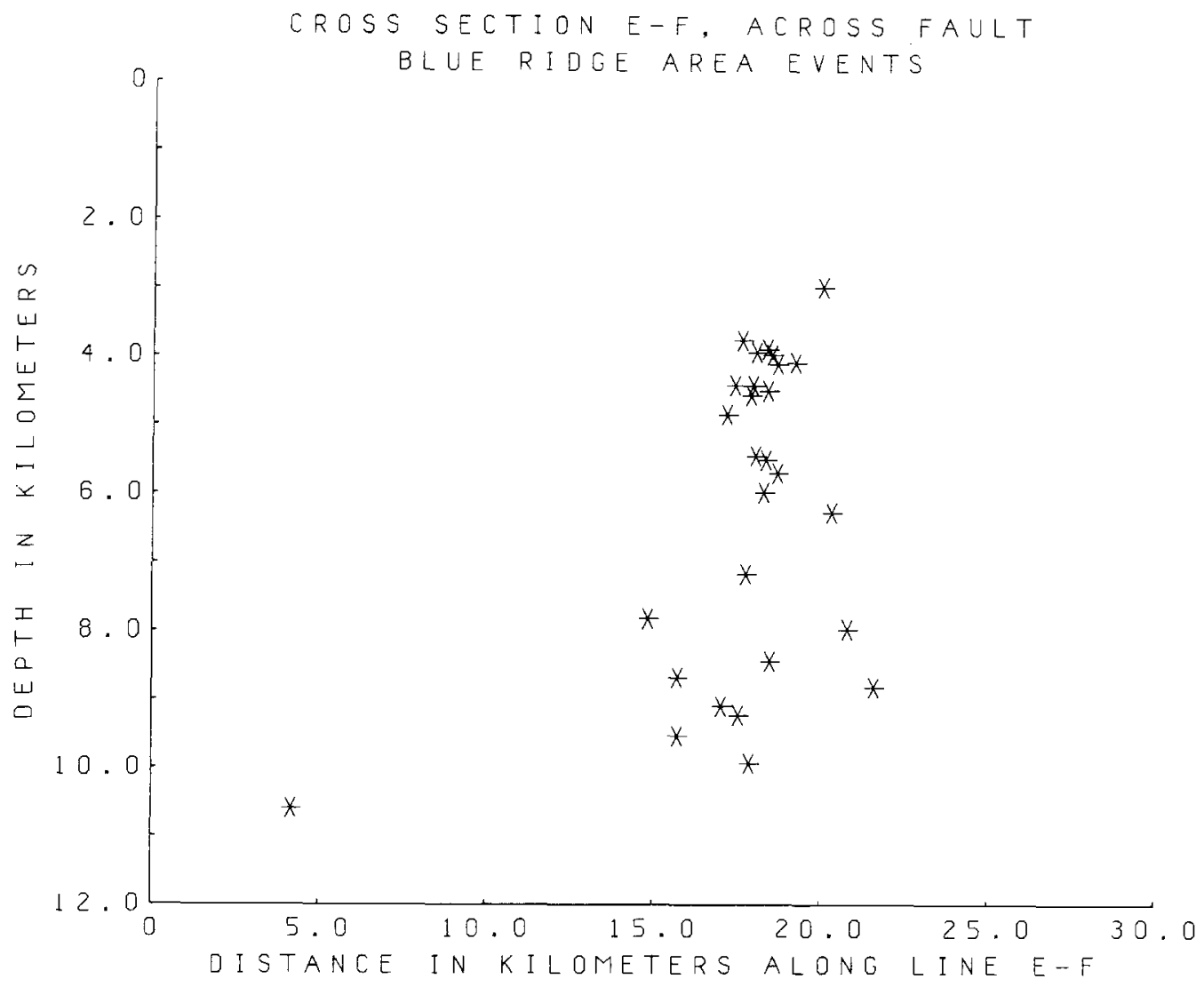

Fig. 8. Cross section taken normal to the San Andreas Fault showing the depth distribution of events in the vicinity of Blue Ridge.

The Brawley swarm, however, was of much shorter duration, lasting only a few days.

In 1979 and the first half of 1980, the rate of seismicity in this area has decreased considerably. Fewer than 20 events were recorded in the Juniper Hills and Blue Ridge areas.

The procedure outlined in this study has been shown to be a viable method of estimating hypocentral parameters of a group of local earthquakes. By using traveltime data from a few regionally well-recorded events, an appropriate local velocity model can be estimated and used to locate other smaller events in the group. The high relative precision of the hypocenters allows examination of the spatial and 


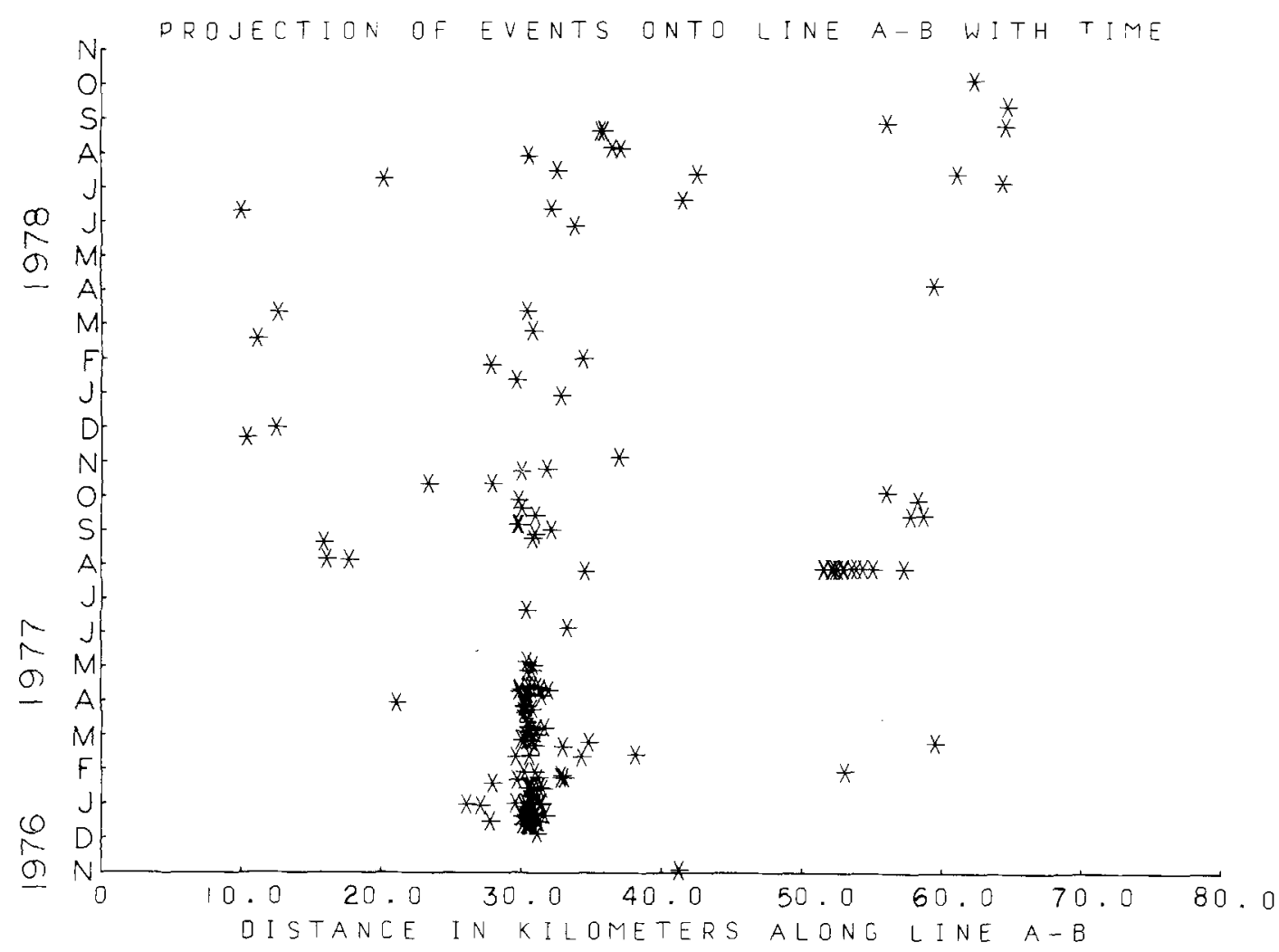

FrG. 9. Time distribution of events projected onto the approximate trace of the San Andreas Fault.

temporal seismicity patterns with greater confidence, so the procedure can be useful in understanding the tectonic significance of local seismicity.

\section{ACKNOWLEDGMENTS}

We would like to thank Dr. L. R. Johnson for his advice and help in the development of the computer program used in this study, and the many useful suggestions he provided throughout the course of the study. We would also like to thank Drs. Hiroo Kanamori and T. V. McEvilly for helpful suggestions, and Mary French for reading the arrival times for all the events used in this study.

This research was partially supported by the Advanced Research Projects Agency of the Department of Defense and was monitored by the Air Force Office of Scientific Research under Contract F49620-79C-0028 (DSD) and by the U.S. Geological Survey Contracts 14-08-0001-16711 and 14-08-001-17631 (KCM).

\section{RefERENCES}

Castle, R. O., J. P. Church, and M. R. Elliott (1976). A seismic uplift in southern California, Science $\mathbf{9 1 2}$, 251.

Crosson, R. S. (1976). Crustal structure modeling of earthquake data I. Simultaneous least squares estimation of hypocenter and velocity parameters, J. Geophys. Res. 81, 3036-3046.

Friedman, M. E., J. H. Whitcomb, C. R. Allen, and J. A. Hileman (1976). Seismicity of the Southern California Region, 1 January 1972 to 31 December 1974, Seismological Laboratory, California Institute of Technology.

Hadley, D. and H. Kanamori (1977). Seismic structure of the Transverse Ranges, California, Bull. Geol. Soc. Am. 88, 1469-1478.

Hileman, J. A., C. R. Allen, and J. M. Nordquist (1973). Seismicity of the Southern California Region, 1 January 1932 to 31 December 1972, Seismological Laboratory, California Institute of Technology.

Hoerl, A. E. and R. W. Kennard (1970). Ridge regression: biased estimation for nonorthogonal problems, Technometrics 12, 55-67. 
Johnson, C. E. and D. M. Hadley (1976). Tectonic implications of the Brawley earthquake swarm, Imperial Valley, California, January 1975, Bull. Seism. Soc. Am. 66, 1133-1144.

Levenberg, K. (1944). A method for the solution of certain nonlinear problems in least squares, Quart. Appl. Math. 2, 164-168.

Marquardt, D. W. (1963). An algorithm for least squares estimation of nonlinear parameters, J. Soc. Indust. Appl. Math. 11, 431-441.

McEvilly, T. V. (1966). Crustal structure estimation within a large scale array, Geophys. J. 11, 13-17.

McNally, K. C., H. Kanamori, and J. C. Pechmann (1978). Earthquake swarm along the San Andreas fault near Palmdale, Southern California, 1976 to 1977 , Science 201, 814-817.

Smith, E. G. C. (1976). Scaling the equations of condition to improve conditioning, Bull. Seism. Soc. Am. 66, 2075-2076.

\section{SEISMOGRAPHIC Station}

Department of Geology and Geophysics

UNIVERSITY OF CALIFORNIA

Berkeley, California 94720 (D.S.D.)
Seismological Laboratory

California Institute of Technology

Pasadena, California 91125 (K.C.M.)

Contribution No. 3348

Manuscript received January 6, 1980 